\title{
Erratum to: Aspects of Integrating Functional Electroceramic Material in Multilayer Thin Films for Image Sensing: Modeling and Experiment
}

\author{
M.A. MATIN,${ }^{1,4,5}$ K. OISHI, ${ }^{2}$ A. KATSUTA,${ }^{2}$ D. AKAI, ${ }^{2}$ K. SAWADA, ${ }^{2,3}$ \\ and M. ISHIDA ${ }^{2,3}$ \\ 1.-Glass and Ceramic Engineering, Bangladesh University of Engineering and Technology, \\ Dhaka 1000, Bangladesh. 2.-Electrical and Electronic Information Engineering, Toyohashi \\ University of Technology, Toyohashi, Aichi 441-8580, Japan. 3.-Electronics-Inspired Interdisci- \\ plinary Research Institute (EIIRIS), Toyohashi University of Technology, Toyohashi 441-8580, \\ Japan. 4.—e-mail: mmatin@gce.buet.ac.bd. 5.—e-mail: matin.md.a@gmail.com
}

\section{Erratum to: Journal of ELECTRONIC MATERIALS DOI 10.1007/s11664-015-3631-x}

On the second page of the original article, in the Residual Stresses section directly below Eq. 1, the corrected text is as follows:

where $\sigma_{i j}$ is the second Piola-Kirchhoff stress tensor, $\varepsilon_{k l}$ the Lagrangian strain tensor, $C_{i j k l}$ is elastic tensor of the solid, $\alpha_{i j}$ denotes the thermal expansion tensor and $\Delta \Theta=\Theta-\Theta_{0}$ represents the temperature difference (where $\Theta_{0}$ is the stress free temperature state and $\Theta$ is the final temperature state). 